\title{
A Case of Melioidosis with Multiple Lung Abscesses, Intrahepatic Cholestasis and Hepatic Encephalopathy
}

\author{
Palangasinghe $\mathrm{DR}^{1 *}$, J ayasekara $\mathrm{S}^{2}$, Hewavithana \\ $\mathrm{J}^{1}$, Dahanayaka $\mathrm{N}^{3}$ and Bodinayake $\mathrm{C}^{3}$ \\ ${ }^{1}$ Senior Registrar in Medicine, University Medical Unit, \\ Teaching Hospital Karapitya, Galle, Sri Lanka \\ ${ }^{2}$ Registrar in Medicine, University Medical Unit, Teaching \\ Hospital Karapitiya, Galle, Sri Lanka \\ ${ }^{3}$ Senior Lecturer in Medicine, Consultant Physician, \\ Faculty of Medicine, University of Ruhuna, Sri Lanka \\ *Correspondling author: Dhammika Randula \\ Palangasinghe, Senior Registrar in Medicine, University \\ Medical Unit, Teaching Hospital Karapitya, Galle, Sri \\ Lanka
}

Received: March 29, 2017; Accepted: April 19, 2017; Published: April 26, 2017

\begin{abstract}
Background: Melioidosis is an infectious disease caused by gram negative bacillus Burkholderia pseudomallei. It is an emerging infection in Sri Lanka and the clinical presentation is not distinctive. We report a case of melioidosis with multiple lung abscesses, intrahepatic cholestasis and hepatic encephalopathy.

Case Presentation: A 56 year old diagnosed patient with type 2 diabetes mellitus, Chronic Inflammatory Demyelinating Polyradiculoneuropathy (CIDP), benign prostatic hypertrophy admitted with high fever with chills and rigors for five days followed by worsening lower limb weakness with acute urine retention. On examination he was emaciated, deeply icteric and febrile, having flapping tremors, with grade 2 weakness, areflexia in lower limbs with a palpable bladder. Three consecutive blood cultures were positive for Burkholderia pseudomallei with multiple bilateral lung abscesses seen on chest radiograph. His magnetic resonance imaging of the lumbosacral spine was normal with evidence of CIDP in the nerve conduction studies. He responded well to intravenous meropenam, oral co-trimoxazole with complete resolution of fever, conscious level and liver functions.
\end{abstract}

Conclusion: Melioidosis is a treatable emerging infection having high morbidity and mortality with wide array of complications including intrahepatic cholestasis. We achieved an excellent response with early antibiotic therapy resulting in complete resolution of fever and liver functions.

Keywords: Melioidosis; Intrahepatic cholestasis; Hepatic encephalopathy; Multiple lung abscesses

\section{Background}

Melioidosis is caused by a saprophytic bacterium, Burkholderia pseudomallei which is gram negative bacillus and is acquired by inoculation or inhalation of contaminated soil/water. Occupational exposure to surface water and mud is a risk factor. Since the presentation of melioidosis range from localized abscesses in skin, soft tissues and lungs to disseminated disease with septicaemia a high index of suspicion is required to diagnose the infection early [1-3].

Melioidosis is frequently reported in South East Asia and Northern Australia [4]. It also occurs in South Pacific, Africa, India, and the Middle East. Sri Lanka lies in the melioidosis belt. Although Sri Lanka is not considered as a country where melioidosis is endemic, an increasing number of cases have been reported recently [5]. Only three cases had been reported between 1927 and 2005 but there were approximately 90 patients detected from 2006 to 2015 in Sri Lanka [4]. The number rose every year, with $70 \%$ detected in $2014 / 15$. Case detection was improved by raising physician, microbiologist and laboratory technician awareness [4]. Cases presented throughout the year with two peaks during monsoons which is probably secondary to increased risk of inhalation related to rainfalls [4]. Still there is a need to increase the awareness regarding this infection among clinicians and clinical microbiologists in addition to improve laboratory facilities if we are to diagnose more cases early followed by appropriate antibiotic therapy since that is the best way to reduce the mortality which was estimated to be about $40 \%$ in 2015 [5].
The clinical presentation is not distinctive and varied. It may be acute or chronic, localized or disseminated. Severe infection is more common in persons with diabetes mellitus, chronic kidney disease, liver disease and alcoholism. Occupational exposure to mud and surface water have made paddy farmers, other cultivators, military personnel, construction workers, livestock farmers and even those who indulge in gardening at higher risk of acquiring the infection.

Isolation of the causative bacterium in the clinical specimens (blood, urine, sputum, or skin-lesion sample) is the gold standard for the definitive diagnosis. Serologic testing alone is inadequate for confirming the diagnosis, especially in endemic regions where the background sero positivity rate can be more than $50 \%[5,6]$.

Melioidosis infection commonly involves the lungs and any organ can be involved with abscess formation. We are reporting a case of melioidosis with multiple lung abscesses and intrahepatic cholestasis with hepatic encephalopathy which fully resolved with appropriate antibiotic treatment.

\section{Case Presentation}

A 56 year old Sri Lankan carpenter from Galle, with a background history of type 2 diabetes mellitus presented with dysuria, lower abdominal pain, straining at micturition, poor stream followed by fever with chills and rigors for five days duration. Urinalysis revealed field full of pus cells with 5-10 red cells in high power field and a urine culture growing $>105$ colony forming units from coliforms sensitive
J Bacteriol Mycol - Volume 4 Issue 2 - 2017

ISSN : 2471-0172 | www.austinpublishing group.com

Palangasinghe et al. (C) All rights are reserved
Citation: Palangasinghe DR, Jayasekara S, Hewavithana J, Dahanayaka N and Bodinayake C. A Case of Melioidosis with Multiple Lung Abscesses, Intrahepatic Cholestasis and Hepatic Encephalopathy. J Bacteriol Mycol. 2017; 4(2): 1048. 


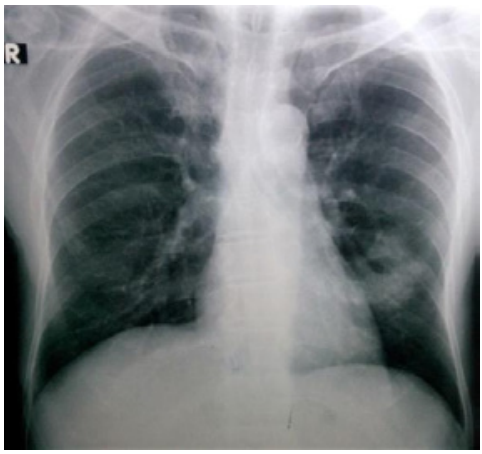

Figure 1: Initial chest radiograph showing left middle zone abscess.

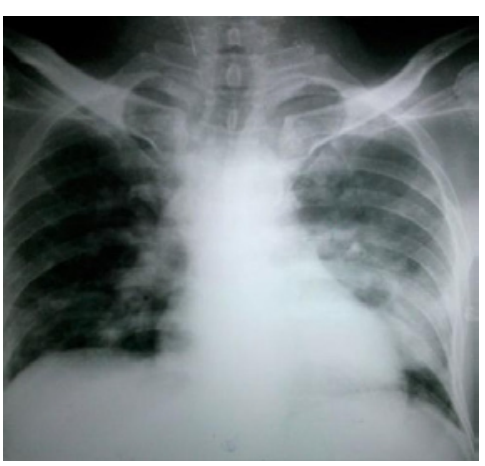

Figure 2: Second chest radiograph showing bilateral multiple abscesses.

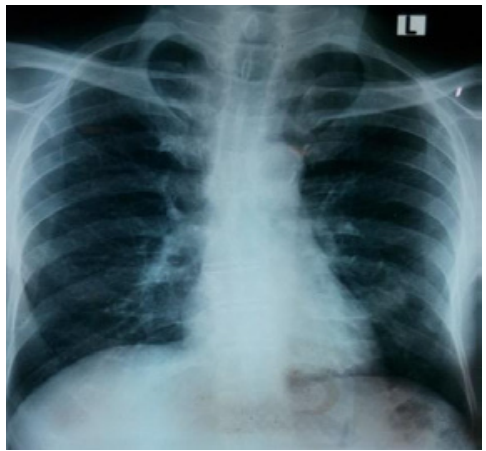

Figure 3: Chest radiograph after two weeks of antibiotics showing resolution of multiple lung abscesses.

for meropenem but resistant for gentamicin. His blood culture did not reveal any growth and he was treated with intravenous meropenam $1 \mathrm{~g}$ three times daily for seven days. His fever and lower urinary tract symptoms resolved on day 4 of the treatment. Ultrasound scan of the abdomen revealed evidence of horseshoe shaped kidney with no evidence of pyelonephritis, perinephric-abscess or hydronephrosis and there was mild prostatomegaly without focal lesions. His serum creatinine value was within normal limits. He was sent home after seven days of antibiotic therapy when he was a febrile for three consecutive days. Five days later fever reappeared with chills and rigors. He again developed similar lower urinary tract symptoms, severe anorexia and nausea followed by acute urine retention. In addition he developed yellowish discoloration of the eyes with inversion of sleep pattern. He also developed worsening lower limb weakness over one week where he became bed bound in background history of gradually worsening lower limb numbness and weakness over last two months. On second admission he was found to be febrile (104 F), drowsy, deeply icteric, having flapping tremors. He was not pale and had no lymphadenopathy. He was tachycardic with pulse rate of 104/min and blood pressure was $110 / 70 \mathrm{mmHg}$. There were no cardiac murmurs. He had coarse crepitations in the mid zone of the left lung. Abdominal examination revealed a palpable bladder with $400 \mathrm{~mL}$ of urine appearing with the catheterization. There was no hepatosplenomegaly. There was grade 2 weakness in the both lower limbs with areflexia with objective stocking type of sensory loss on both feet.

His haematological investigations revealed neutrophil leukocytosis $(18.95 \times 103 / \mu \mathrm{l} ; \mathrm{N}-88 \%)$, elevated inflammatory markers (CRP $333 \mathrm{mg} / \mathrm{dl}$; ESR $108 \mathrm{~mm} 1^{\text {st }}$ hour). Initial chest radiograph showed left mid zone cavitating lesion. However repeat chest radiograph performed five days later showed bilateral multiple cavitating lesions compatible with multiple lung abscesses. There were no vegetations in the 2D echocardiogram (Figure 1-3).

Liver profile revealed aspartate transaminase $27 \mathrm{U} / \mathrm{L}$, alanine transaminase $23 \mathrm{U} / \mathrm{L}$, total bilirubin $40 \mu \mathrm{mol} / \mathrm{L}$, direct bilirubin 31.5 $\mu \mathrm{mol} / \mathrm{L}$, ALP $277 \mathrm{U} / \mathrm{L}$, gamma Glutamyl transferase $128 \mathrm{U} / \mathrm{L}$ and prothrombin time international normalized ratio of 1.3 .

Ultra sound scan of the abdomen showed intrahepatic duct dilatation with sludge in the gallbladder. Common bile duct was normal and there were no gallstones. There was no evidence of pyelonephritis or obstructive uropathy. Urinalysis on second admission revealed 60-70 pus cells, positive bile salts with no growth in the urine culture. Burkholderia pseudomallei was isolated in three consecutive blood cultures which were performed on admission. Nerve conduction studies were suggestive of chronic inflammatory demyelinating polyradiculoneuropathy. Magnetic resonance imaging of lumbosacral spine was normal.

Intravenous meropenam $1 \mathrm{~g} 8$ hourly, oral trimethoprimsulfamethoxazole $1440 \mathrm{mg}$ twice daily commenced once blood cultures were positive for gram negative bacilli and he showed excellent response to medical therapy. Fever completely resolved over one week and he regained full consciousness without any residual drowsiness. His weakness improved to baseline level and he could walk without support on day 16 of the second admission (Table 1).

\section{Discussion}

Meliodosis is an important emerging infection in Sri Lanka with a wide range of clinical manifestations ranging from localized abscesses to septicaemia with multiple organ involvement $[1,2]$. Inhalation and direct inoculation are important routes of infection. Paddy farmers, other cultivators, military personnel are at a higher risk because of frequent exposure to mud and surface water [3,4]. Increase incidence related to two monsoons probably secondary to increase in inhalation related to rains $[3,4,5]$.

Our patient had diabetes mellitus and consumption of alcohol which are well known predisposing conditions for melioidosis. Being a carpenter with frequent visits to forests for wood cutting increased the risk of exposure to bacteria. Pulmonary abscesses with prolonged 
Table 1: Summary of haematological investigations during the course of the illness.

\begin{tabular}{|c|c|c|c|c|c|c|}
\hline Investigation & & $08 / 12$ & $14 / 12$ & $17 / 12$ & $23 / 12$ & $28 / 12$ \\
\hline \multirow[t]{3}{*}{$\begin{array}{c}\text { Full Blood } \\
\text { Count }\end{array}$} & $\begin{array}{l}\text { WBC }\left(\times 10^{3} / \mu \mathrm{l}\right) \\
\text { Neutrophil } \%\end{array}$ & $\begin{array}{l}18.9 \\
88 \%\end{array}$ & $\begin{array}{l}18.7 \\
87 \%\end{array}$ & $\begin{array}{c}21 \\
84 \%\end{array}$ & $\begin{array}{c}12.37 \\
82 \%\end{array}$ & $\begin{array}{c}10 \\
78 \%\end{array}$ \\
\hline & Hemoglobin (g/dl) & 11.4 & 9.9 & 8 & 8 & 9 \\
\hline & Platelets $\left(\times 10^{3} / \mu \mathrm{l}\right)$ & 262 & 304 & 387 & 755 & 560 \\
\hline $\begin{array}{c}\text { ESR } \\
\left(\mathrm{mm} / 1^{\text {st }} \text { hour }\right)\end{array}$ & & 108 & & 110 & 32 & 30 \\
\hline CRP (mg/dl) & & 333 & 204 & & 16 & 8 \\
\hline \multirow{7}{*}{ Liver profile } & $\begin{array}{l}\text { Total protein }(\mathrm{g} / \mathrm{dl}) \\
\text { Albumin }\end{array}$ & $\begin{array}{l}53 \\
20\end{array}$ & & $\begin{array}{l}54 \\
22\end{array}$ & $\begin{array}{l}55 \\
23\end{array}$ & $\begin{array}{l}58 \\
30\end{array}$ \\
\hline & $\operatorname{ALT}(\mathrm{U} / \mathrm{L})$ & 23 & & 16 & 11 & 18 \\
\hline & AST (U/L) & 27 & & 20 & 12 & 14 \\
\hline & $\begin{array}{c}\text { Total Bilirubin } \\
(\mu \mathrm{mol} / \mathrm{l}) \\
\text { Direct }\end{array}$ & $\begin{array}{c}40 \\
31.5\end{array}$ & & $\begin{array}{c}18 \\
13.6\end{array}$ & $\begin{array}{l}10 \\
8.3\end{array}$ & $\begin{array}{l}8 \\
2\end{array}$ \\
\hline & ALP (U/L) & 277 & & 283 & 169 & 104 \\
\hline & GGT(U/L) & 128 & & 120 & 106 & 80 \\
\hline & PT/INR & 1.12 & & 1.3 & 1.1 & 1.1 \\
\hline
\end{tabular}

fever which occurred in our patient, is a well known presentation in melioidosis [7]. In most patients exposure to wet soil and surface water with underlying predisposing conditions such as diabetes mellitus was demonstrated as in our patient [7]. It is an important differential diagnosis for sputum negative pulmonary tuberculosis in the tropical setting [7]. Early identification and initiation of appropriate antibiotic treatment is the key to reduce the morbidity and mortality. Our patient had intrahepatic cholestasis as evident by deep icterus, direct hyperbillirubinaemia, elevated alkaline phosphatase, gamma Glutamyl transferase, with normal transaminases, dilated intrahepatic ducts and normal common bile duct on ultrasound scan. These were accompanied with inversion of sleep pattern and flapping tremors suggestive hepatic encephalopathy. The onset of intrahepatic cholestasis occurred in the middle of the infection and complete resolution with the antibiotic treatment made us assuming the direct correlation of intrahepatic cholestasis to melioidosis. Cholestasis is a well known complication of sepsis related to bacterial infection among children and it has also been described with adults. In fact gram negative bacterial infections with sepsis with cholestasis accounted for one third of neonatal jaundice [7]. Most commonly associated organism was Escherichia coli and various infections associated were pneumonia, pyelonephritis and meningitis. Several mechanisms were thought to be responsible for the development of cholestasis with gram negative septicaemia without direct liver involvement of bacteria. One of them was cytokines in response to infection interfering with the transport systems in the sinusoids and biliary canaliculi [8]. To the best of our knowledge intrahepatic cholestasis was not previously described with melioidosis. Complete resolution of cholestasis and hepatic encephalopathy was seen after two weeks of appropriate antibiotic treatment in our patient. Since he had multiple lung abscesses and was critically ill initially we treated him with meropenem and Co- trimoxazole for 4 weeks in the intensive phase [11]. He was commenced on co-trimoxazole and doxycycline for the eradication phase which we are planning to continue for at least 3 months in order to minimize the risk of relapse $[11,12]$.

The lower limb weakness with areflexia without a sensory level associated with the infection also resolved with the treatment with antibiotics. The most plausible explanation for that was the worsening of preexisting CIDP which was evident in the nerve conduction studies. This was different to the development of Guillain-Barre Syndrome which was previously reported as a rare complication of melioidosis $[9,10]$. Normal MRI spine excluded the cord compression related to abscess formation [13].

Our report added to the literature of an important emerging infection in Sri Lanka with high morbidity and mortality. It also highlights the possible occurrence of intrahepatic cholestasis with hepatic encephalopathy with excellent recovery with the appropriate antibiotic treatment.

\section{Consent}

Written informed consent was obtained from the patient for the publication of this case report and any accompanying images.

\section{References}

1. White NJ. Melioidosis. Lancet. 2003; 361: 1715-1722.

2. Leelarasamee A, Bovornkitti S. Melioidosis: Review and update. Reviews of Infectious Diseases. 1989; 2: 413-425.

3. Jayasekara K, Perera S, Wijesundere A. Fatal Burkholderia pseudomallei septicaemia. Ceylon Medical Journal. 2006; 51: 69-70.

4. Nationwide epidemiology of melioidosis - F1000Research. Sri Lanka.

5. Weekly epidemiology report. Sri Lanka.

6. Currie BJ, Ward L, Cheng AC. The epidemiology and clinical spectrum of melioidosis: 540 cases from the 20 year darwin prospective study. PloS Negl Trop Dis. 2010, 4: 900

7. Rammaert B, Beauté J, Borand L, Hem S, Buchy P, Goyet S, et al. Pulmonary melioidosis in Cambodia: A prospective study. BMC Infectious Diseases. 2011. 11:126. DOI: 10.1186/1471-2334-11-126. (c) Rammaert et al; licensee BioMed Central Ltd. 2011.

8. Zimmerman H. Jaundice due to bacterial infection. Gastroenterology. 1979; 77: 362-374. PubMed.

9. Pirovino M, Meister F, Rubli E, Karlaganis G. Preserved cytosolic and synthetic liver function in jaundice of severe extrahepatic infection. Gastroenterology. 1989; 96: 1589-1595. PubMed.

10. Krovvidi R, Mridula RK, Jabeen SA, Meena AK. Guillain Barre syndrome as a manifestation of neurological melioidosis. Indian Acad Neurol. 2013; 16: 681-683.

11. Wijekoon PW, Bandara KA, Kailainathan A, Chandrasiri NS, Hapuarachchi CT. Guillaine-barre syndrome; a rare complication of melioidosis. a case report. BMC Infect Dis. 2016; 16: 388.

12. Chaowagul W. Recent advances in the treatment of severe melioidosis. Acta Trop. 2000; 74: 133.

13. Rajchanuvong A, Chaowagul W, Suputtamongkol $Y$, Smith MD, Dance DA, White NJ. A prospective comparison of co-amoxiclav and the combination of chloramphenicol, doxycycline, and co-trimoxazole for the oral maintenance treatment of melioidosis. Trans R Soc Trop Med Hyg. 1995; 89: 546.

\footnotetext{
J Bacteriol Mycol - Volume 4 Issue 2 - 2017 ISSN : 2471-0172 | www.austinpublishinggroup.com Palangasinghe et al. (C) All rights are reserved
}

Citation: Palangasinghe DR, Jayasekara S, Hewavithana J, Dahanayaka N and Bodinayake C. A Case of Melioidosis with Multiple Lung Abscesses, Intrahepatic Cholestasis and Hepatic Encephalopathy. J Bacteriol Mycol. 2017; 4(2): 1048 